[0212-7199 (2007) 24: 3; pp 129-131] ANALES DE MEDICINA INTERNA Copyright (@) 2007 ARAN EDICIONES, S.L.

AN. MED. INTERNA (Madrid) Vol. 24, N. ${ }^{\circ} 3$, pp. 129-131, 2007

\section{Síndrome intermedio en el transcurso de una intoxicación por organofosforados a pesar de una infusión continua de pralidoxima}

\author{
M. PERAYRE BADIA, E. LEIVA BADOSA, L. PASTÓ CARDONA, \\ R. JÓDAR MASSANÉS
}

Servicio de Farmacia. Hospital Universitari de Bellvitge. L'Hospitalet de Llobregat. Barcelona

\begin{abstract}
RESUMEN
En las intoxicaciones agudas por insecticidas organofosforados se produce un síndrome colinérgico debido a un aumento de la aceticolina a nivel sináptico, como consecuencia de una inhibición de la acetilcolinesterasa. En algunos casos, tras la resolución de la sintomatología colinérgica, se presenta el síndrome intermedio, caracterizado por una parálisis de la musculatura proximal y diafragmática. Describimos el caso de un paciente de 67 años que desarrolla un síndrome intermedio tras la ingestión de una cantidad desconocida de oxidemetón-metilo en un intento de autolisis, a pesar de la infusión continuada de pralidoxima a dosis altas. Diversas hipótesis tratan de explicar la etiología del síndrome intermedio (disfunción de la unión neuromuscular, instauración de un tratamiento inadecuado o de inicio tardío con oximas, entre otras). Asimismo, su manifestación se ve condicionada por la persistencia del insecticida organofosforado en el organismo, su estructura química o el tiempo transcurrido entre la ingestión del tóxico y la administración del antídoto.
\end{abstract}

PALABRAS CLAVE: Organofosforados. Síndrome intermedio. Intoxicación. Pralidoxima. Oxidemetón-metilo.
INTERMEDIATE SYNDROME AFTER ORGANOPHOSPHATE POISONING DESPITE CONTINUOUS INFUSION OF PRALIDOXIME

\begin{abstract}
Acute organophosphate poisoning leads to a cholinergic crisis secondary to an acetylcholine rise, developed by an acetylcholinesterase inhibition. In some cases, after the resolution of the initial cholinergic signs and symptoms, an intermediate syndrome occurs, characterized by a delayed development of proximal and diaphragmatic muscle paralysis. We describe a case of a 67-year-old man who developed an intermediate syndrome after oxydemeton-metryl ingestion in a suicide attempt, despite a continuous pralidoxime infusion. Several hypotheses have been developed to explain the aetiology of this intermediate syndrome (neuromuscular junction dysfunction, inadequate poisoning treatment, late beginning of the oxime administration, etc). Intermediate syndrome manifestation will depend on the organophosphate's organism persistence and its chemical structure, and also on the time elapsed between the poisoning and the antidote administration.
\end{abstract}

KEY WORDS: Organophosphates. Intermediate syndrome. Poisoning. Pralidoxime. Oxydemeton methyl.

Perayre Badia M, Leiva Badosa E, Pastó Cardona L, Jódar Massanés R. Síndrome intermedio en el transcurso de una intoxicación por organofosforados a pesar de una infusión continua de pralidoxima. An Med Interna (Madrid) 2007; 24: 129-131.

\section{INTRODUCCIÓN}

En las intoxicaciones agudas por insecticidas organofosforados $(\mathrm{OP})$ se produce una inhibición de la acetilcolinesterasa (ACE), que se traduce en un aumento de la acetilcolina en las uniones sinápticas. La aparición de los síntomas está en función de la vía de exposición. Aunque se absorben rápidamente por todas las vías (respiratoria, gastrointestinal, ocular y dérmica), la vía inhalatoria es la más rápida (1).

Las intoxicaciones agudas por OP pueden manifestarse en tres entidades clínicas distintas que se suceden en el tiempo: a) una crisis colinérgica aguda, que aparece en pocos minutos u horas post-intoxicación; b) un síndrome intermedio (SI), que aparece a las 48-72 horas después de la intoxicación; y c) una polineuropatía tardía inducida por OP (2).

El SI, descrito en 1987, se inicia una vez se han resuelto los síntomas de la crisis colinérgica inicial y se manifiesta con debilidad de la musculatura respiratoria y proximal, y parálisis craneal. Los organofosforados asociados mayoritariamente con el SI son el fentión, el dimetoato, el diazinón, el monocrotofós, el metamidofós y metilo-paratión, y raramente el malatión $(3,4)$.

En el presente trabajo se describe el caso de una intoxicación por oxidemetón-metilo, que desarrolló un SI, a pesar de la infusión continua de pralidoxima a altas dosis.

Trabajo aceptado: 17 de octubre de 2006 


\section{CASO APORTADO}

Presentamos el caso clínico de un varón de 67 años, con antecedentes de enolismo moderado-severo, hipertensión arterial y cardiopatía isquémica, que es trasladado al Servicio de Urgencias procedente de otro centro donde ha acudido 4 horas post-ingesta de un OP (Metasystox ${ }^{\circledast}$ : oxidemetón-metilo) en un intento de autolisis. En el centro de procedencia se le ha practicado un lavado gástrico y se le ha administrado una dosis de carbón activado.

A su llegada a urgencias el paciente está consciente y orientado, hipotenso y bradicárdico, con claras manifestaciones muscarínicas: sudoración profusa, sialorrea, abundante secreción lacrimal, y dolor difuso de tipo cólico con náuseas y vómitos. A nivel nicotínico, no presenta déficit motor pero sí alguna fasciculación perceptible en ambos muslos. Durante su paso por el Servicio de Urgencias el paciente presenta mioclonías generalizadas, miosis y desaturación con cianosis periférica, probablemente secundaria a las fasciculaciones de la musculatura periférica y del diafragma.

Ante la sintomatología, se inicia tratamiento conjunto con atropina y pralidoxima ( $1 \mathrm{~g}$ en 30 minutos), consiguiendo una mejora de los síntomas periféricos, con disminución del número de fasciculaciones y desaparición de los cólicos abdominales. Asimismo, se administra una segunda dosis de $25 \mathrm{~g}$ de carbón activado y un bolus de diazepam intravenoso para el control de las convulsiones.

Ante la refractariedad de las mismas, se procede a intubación orotraqueal para proteger la vía aérea y a la instauración de un tratamiento con pralidoxima (12 g/día) y atropina ( $20 \mathrm{mg} /$ día $)$ ambas en perfusión continua. En estas condiciones es ingresado en la Unidad de Vigilancia Intensiva (UVI), donde permanece 12 días. Durante los 10 primeros días post-intoxicación se mantiene con el tratamiento instaurado en urgencias, según las dosis especificadas en la tabla I.

Hasta el día 6 post-intoxicación, el paciente presenta miosis y peristaltismo elevado, y a partir de este día manifiesta midriasis y tolerancia dificultada a la nutrición enteral como consecuencia de la ausencia de peristaltismo. Éste mejora al disminuir la dosis de atropina. Sin embargo, la midriasis reactiva persiste hasta el alta a planta. Otra sintomatología del paciente, característica de las intoxicaciones por OP, son mioclonías generalizadas y debilidad muscular torácica. Dicha debilidad podría asimismo ser consecuencia de la sedación a la que está sometido por su estado de agitación. Hacia el día 5 postintoxicación son perceptibles mioclonías faciales aisladas, todavía en un contexto de clínica neurológica.

La recuperación progresiva de la fuerza de las extremidades empieza el día 6 post-intoxicación, siendo completa el día 9. Sin embargo, entre estos días, aun bajo tratamiento con pralidoxima en perfusión continua, el paciente desarrolla un SI con debilidad muscular proximal y facial. Ésta condiciona la hipoventilación por fatiga muscular.

En cuanto a la clínica respiratoria, crítica en el intoxicado por $\mathrm{OP}$, presenta un episodio de insuficiencia respiratoria inicial, corregida posteriormente al mantener al paciente bajo ventilación mecánica (VM) hasta 5 días después de la intoxicación. A partir de este día, se inician desconexiones graduales de la VM, ante las cuales el paciente tiende a la hipoventilación por fatiga muscular. La mejoría de la función respiratoria, aunque dificultada por una traqueobron- quiolitis nosocomial por $H$. influenzae, se manifiesta hacia el día 8 post-intoxicación. A pesar de la agitación psicomotriz, tolera desconexiones de la VM que permiten la extubación un día después de iniciadas. No obstante, persiste una tendencia a la hipoventilación a lo largo de su estancia en la UVI.

Como biomarcador de exposición al tóxico se valoran los niveles de colinesterasa plasmática, cuya primera determinación, realizada a las 16 horas post-ingesta, mostró un nivel de 13 ukat/L (intervalo de referencia: 89-215 ukat/L). Su evolución puede apreciarse en la figura 1.

\section{Colinesterasa plasmática}

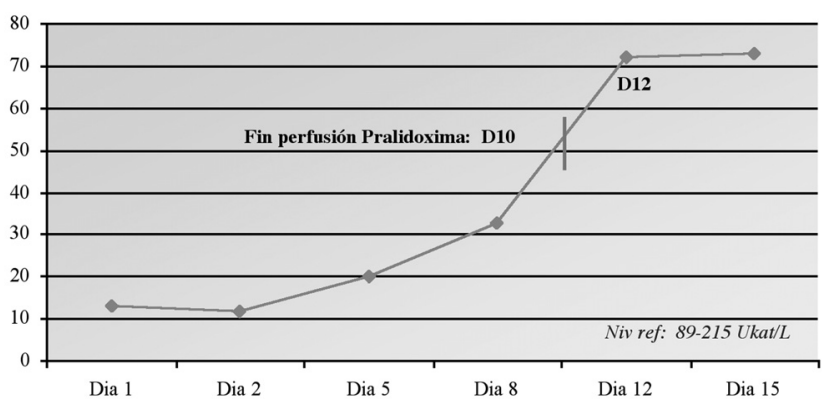

Fig. 1. Evolución de los niveles de colinesterasa plasmática.

\section{DISCUSIÓN}

La actitud terapéutica a tomar ante una intoxicación por OP comprende fundamentalmente: la descontaminación gastrointestinal con lavado gástrico y/o carbón activado (controvertido en ingestas notables, donde la persistencia de náuseas y vómitos lo puede contraindicar) o la descontaminación de otras vías de contacto con el OP (ropa, piel, ojos) (5); el establecimiento de terapia de soporte (protección de la vía aérea, monitorización cardio-pulmonar); la utilización de atropina (terapia anticolinérgica) y de oximas (antídoto que reactivará la ACE inhibida) (1).

La etiología del SI es desconocida, pero existen numerosas hipótesis que pretenden estudiar las causas de dicha entidad. Algunas investigaciones sobre la fisiopatología del SI muestran la existencia de una disfunción de la unión neuromuscular, posiblemente desencadenada por la inhibición continuada de la acetilcolinesterasa (6). La debilidad muscular podría ser consecuencia de la prolongada estimulación colinérgica, desembocando en una desensibilización de los receptores colinérgicos (2).

La asociación del SI con un tratamiento inadecuado o un inicio tardío con oximas, después del envejecimiento de la

TABLA I

PAUTAS POSOLÓGICAS DEL TRATAMIENTO DE LA INTOXICACIÓN

\begin{tabular}{|c|c|c|c|c|c|}
\hline Días post-intoxicación & $\begin{array}{lllll}1 & 2 & 3 & 4 & 5 \\
\end{array}$ & 6 & 7 & 8 & 10 \\
\hline Perfusión continua de Pralidoxima & & $\begin{array}{l}500 \mathrm{mg} / \mathrm{h} \\
\text { (12 g/día) }\end{array}$ & & & $\begin{array}{c}250 \mathrm{mg} / \mathrm{h} \\
(6 \mathrm{~g} / \mathrm{día})\end{array}$ \\
\hline Perfusión continua Atropina & $\begin{array}{c}3,5 \mathrm{mg} / \mathrm{h} \\
(84 \mathrm{mg} / \mathrm{día})\end{array}$ & $\begin{array}{c}1,7 \mathrm{mh} / \mathrm{h} \\
(40 \mathrm{mg} / \mathrm{día})\end{array}$ & $\begin{array}{c}1 \mathrm{mg} / \mathrm{h} \\
(24 \mathrm{mg} / \mathrm{día})\end{array}$ & $\begin{array}{c}1,8 \mathrm{mg} / \mathrm{h} \\
\text { (44 mg/día) }\end{array}$ & $\begin{array}{c}1 \mathrm{mg} / \mathrm{h} \\
(24 \mathrm{mg} / \mathrm{día})\end{array}$ \\
\hline
\end{tabular}


ACE, avala esta hipótesis. Johnson et al, en un estudio en el que evaluaban dos pautas de pralidoxima en pacientes intoxicados por OP, determinaron una reducción significativa en la incidencia del SI si la pralidoxima se administraba dentro de las 12 horas post-ingestión del tóxico $(7,8)$. Sin embargo, en el caso descrito, el paciente desarrolla un SI entre los días 6 y 9 post-intoxicación, a pesar del inicio de la administración de pralidoxima dentro de las 12 horas post-intoxicación.

Otros factores determinantes de la aparición del SI son la gravedad de la intoxicación (dosis absorbida) y la cinética del OP (lipofilia). En efecto, el SI se ha asociado a determinados OP muy potentes (dimetoato, monocrotofós, metamidofós) o altamente lipófilos (fentión, clorfentión). El papel de la lipofilia del OP está directamente relacionado con la persistencia del tóxico en el organismo, como muestran algunos estudios de intoxicaciones por vía oral que describen una persistencia de OP en fluidos gástricos durante varios días post-ingestión, a pesar de repetidos lavados gástricos $(9,10)$. Concretamente, en la presente intoxicación se trata de una ingesta abundante de oxidemetón-metilo, compuesto dimetilado y altamente lipófilo (11), que se distribuye rápidamente en el organismo, acumulándose en tejido adiposo. El tóxico irá retornando al torrente sanguíneo desde estos depósitos grasos, alargando así su tiempo de permanencia en el organismo.

Otras teorías alternativas que tratan de explicar la etiología del SI se basan en un posible desarrollo de miopatia necrotizante, una depleción de calcio en los miocitos secundaria a la estimulación prolongada del receptor post-sináptico de la acetilcolina $(10,12)$ o un daño oxidativo en las membranas musculares inducido por la acetilcolina durante la fase aguda de la intoxicación (13).

Los marcadores bioquímicos para valorar la evolución de una intoxicación por OP son la determinación de la actividad de la acetilcolinesterasa eritrocítica y la colinesterasa plasmática o pseudocolinesterasa. La primera es un indicador indirecto de la actividad acetilcolinesterasa del cerebro, tejido periférico y músculo esquelético, constituyendo un marcador más fiable que la pseudocolinesterasa, cuya función endógena es desconocida. Sin embargo, la actividad de esta última se recupera más rápidamente que la de la acetilcolinesterasa eritrocítica. Por este motivo, la colinesterasa plasmática es un marcador más idóneo para monitorizar la evolución de un paciente intoxicado por OP. En relación con el SI, se ha observado una depleción prolongada de los niveles de los dos marcadores durante la aparición del mismo (10).

Puesto que la pseudocolinesterasa es una proteína de síntesis hepática, sus niveles estarán diminuidos en situaciones de malnutrición y en enfermedades hepáticas. Algunos fármacos como succinilcolina y lidocaína pueden también reducirlos (1). El paciente descrito presenta antecedentes de enolismo que podrían explicar el hecho de que su colinesterasa plasmática basal, una vez revertida la inhibición, se sitúe por debajo del umbral inferior del intervalo de referencia.

\section{Bibliografía}

1. Kwong TC. Organophosphate pesticides: Biochemistry and Clinical Toxicology. Ther Drug Monit 2002; 24: 144-149.

2. Costa LG. Current issues in organophosphate toxicology. Clin Chim Acta 2006; 366 (1-2): 1-13.

3. Benslama A, Moutaouakkil S, Charra B, Menebhi L. Le syndrome intermédiaire des intoxications aiguës par les insecticides organophosphorés. Ann Fr Anesth Reanim 2004; 23: 353-356.

4. Dandapani M, Zachariah A, Kavitha MR, Jeyaseelan L, Oommen A. Oxidative damage in intermedious syndrome of acute organophosphorous poisining. Indian J Med Res 2003; 117: 253-259.

5. Reigart JR, Roberts JR. Recognition and Management of Pesticide Poisonings. 5th edition, 1999. http: //www.epa.gov/pesticides/safety/healthcare

6. DeBleecker JL. The intermediate síndrome in organophosphate poisoning: An overview of experimental and clinical observations. J Toxicol Clin Toxicol 1995; 33: 683-686.

7. Peter JV, Moran JL, Graham P. Oxime therapy and outcome in human organophasphate poisoning: An evaluation using meta-analytic techniques. Crit Care Med 2006; 34: 502-510.
8. Johnson S, Peter JV, Thomas K, et al. Evaluation of two treatment regimens of pralidoxime ( $1 \mathrm{mg}$ single bolus vs $12 \mathrm{mg}$ infusion) in the management of organophosphorus poisoning. A randomized, double blind controlled clinical trial. J Assoc Physicians India 1996; 44: 529531.

9. Sudakin DL, Mullins ME, Horowitz BZ, et al. Intermediate syndrome after malathion ingestion despite continuous infusion of pralidoxime. $\mathrm{J}$ Toxicol Clin Toxicol 2000; 38: 47-50.

10. Mattingly JE, Sullivan JE, Spiller HA, et al. Intermediate syndrome after exposure to chlorpyrifos in a 16-month-old girl. J Emerg Med 2003; 25: 379-381.

11. Ficha de datos de seguridad de Metasystox: A066242/08. Bayer. 6 de agosto de 2001 .

12. Fengsheng $\mathrm{H}$, Haibing $\mathrm{X}$, Fukuang $\mathrm{Q}$, et al. Intermediate myasthenia syndrome following acute organophosphates poisoning-a analysis of 21 cases. Hum EXP toxicol 1998; 17: 40-45.

13. Dandapani M, Zachariah A, Kavitha MR, et al. Oxidative damage in intermediate syndrome of acute organophosphorous poisoning. Indian J Med Res 2003; 117: 253-259. 